\title{
Preoperative albumin to globulin ratio predicts survival in clear cell renal cell carcinoma patients
}

\author{
Zhen Chen ${ }^{1, *}$, Yingjie Shao ${ }^{2, *}$, Hongwei Yao ${ }^{1}$, Qianfeng Zhuang ${ }^{1}$, Kun Wang ${ }^{1}$, \\ Zhaoyu Xing ${ }^{1}$, Xianlin $X_{\mathbf{u}^{3, *},}$ Xiaozhou $\mathrm{He}^{1, *}$ and Renfang $\mathrm{Xu}^{1, *}$ \\ ${ }^{1}$ Department of Urology, The Third Affiliated Hospital of Soochow University, Changzhou, P.R. China \\ ${ }^{2}$ Department of Radiation Oncology, The Third Affiliated Hospital of Soochow University, Changzhou, P.R. China \\ ${ }^{3}$ Department of Urology, Sir Run Run Shaw Hospital, Third Affiliated Hospital, Nanjing Medical University, Nanjing, Jiangsu, \\ P.R. China \\ *These authors contributed equally to this work
}

Correspondence to: Xianlin Xu, email: xuxianlincz@163.com Xiaozhou He, email: 151363683@qq.com

Renfang Xu, email: xurenfangcz@163.com

Keywords: albumin, globulin, nomogram, prognosis, renal cell carcinoma

Received: February 22, $2016 \quad$ Accepted: January 16, $2017 \quad$ Published: February 07, 2017

Copyright: Chen et al. This is an open-access article distributed under the terms of the Creative Commons Attribution License 3.0 (CC BY 3.0), which permits unrestricted use, distribution, and reproduction in any medium, provided the original author and source are credited.

\section{ABSTRACT}

In this retrospective analysis, we evaluated associations between albumin to globulin ratio (AGR), clinicopathological characteristics, and survival in 592 patients with localized or locally advanced clear cell renal cell carcinoma (CCRCC) prior to nephrectomy. We found that low AGR was associated with more aggressive tumor behavior; patients with low AGR had poorer overall survival (OS) and cancer-specific survival (CSS) in Kaplan-Meier survival analyses both before and after propensity score matching, which was used to compensate for differences in baseline clinicopathological characteristics. AGR was an independent prognostic factor for both OS (HR: 6.799; 95\% CI: 3.215-14.377; $P<0.001$ ) and CSS (HR: 8.806; 95\% CI: 3.891-19.928; $P<0.001$ ), and its prognostic value was higher than that of other established inflammation-based prognostic scores. When AGR was incorporated into a prognostic model that included T stage, neutrophil to lymphocyte ratio (NLR), and monocyte to lymphocyte ratio (MLR), the resulting nomogram predicted 3- and 5-year OS in the patients more accurately than when AGR was not included. In conclusion, AGR may be particularly useful for improving clinical outcome predictions for patients with localized or locally advanced CCRCC.

\section{INTRODUCTION}

Renal cell carcinoma (RCC) accounts for $2 \%-3 \%$ of adult malignancies, and is the second most common type of urogenital neoplasm after bladder cancer [1]. Clear cell renal cell carcinoma (CCRCC) is the most common pathological subtype, accounting for $70 \%-80 \%$ of all RCC cases. As RCC is not sensitive to chemoradiotherapy, radical nephrectomy remains the most promising treatment for patients with localized or locally advanced RCC. However, postoperative recurrence rates of $20 \%-30 \%$ are observed after this treatment [2]. In order to evaluate postoperative risks and to improve individualized treatment, several prognostic models based on clinicopathological characteristics, such as TNM stage [3] and Fuhrman grade [4], have been established to predict outcomes in RCC patients. However, prognostic models based on clinicopathological characteristics alone are less effective for patients with localized or locally advanced CCRCC; a novel prognostic model is urgently needed to improve outcome predictions for these patients.

Albumin and globulin are major serum proteins, and low albumin and high globulin levels reflect not only malnutrition, but also a chronic inflammatory state in the body $[5,6]$. The albumin to globulin ratio (AGR) better reflects the nutritional and inflammatory state by combining these two indicators in one measure. Inflammatory reactions associated with malnutrition greatly reduce immune system function, alter the tumor microenvironment, promote tumor growth and metastasis, and negatively impact prognosis in cancer patients [7, 8]. Accordingly, AGR seems to be indicative of disease 
progress in cancer. Several studies have confirmed that AGR is associated with prognosis in breast [9], colorectal [10], nasopharyngeal [11], and lung cancers [12]. However, the association between AGR and prognosis in RCC patients has not yet been examined.

In this paper, we evaluated the prognostic value of preoperative AGR and compared its value to that of other inflammation-based prognostic scores. Furthermore, we identified a nomogram that combines inflammation-based prognostic scores with clinicopathological characteristics to accurately predict 3 - and 5-year overall survival (OS) in patients with localized or locally advanced CCRCC after radical or partial nephrectomy.

\section{RESULTS}

\section{Patient characteristics}

The clinicopathologic characteristics of patients in the primary and validation cohorts are shown in Table 1. The primary cohort consisted of a total of 258 men $(62.0 \%)$ and 158 women $(38.0 \%)$ with localized or locally advanced CCRCC who underwent radical or partial nephrectomy. $154(37.0 \%)$ of these patients were older than 60 years, and $262(63.0 \%)$ were younger; the median age was 56.3 (range 24-80) years. The median follow-up period was 69.2 (range 1-151) months; at final follow-up, $51(12.3 \%)$ patients had died, and $365(87.7 \%)$ were alive.

The validation cohort included 115 men $(65.3 \%)$ and 61 women $(34.7 \%) ; 71(40.3 \%)$ of these patients were older than 60 years. The median follow-up period was 42.3 (range 3-50) months. At final follow-up, 23 (13.1\%) patients had died, and $153(86.9 \%)$ were alive.

\section{Cut-off values for continuous variables}

The cut-off values for all continuous variables are shown in Table 1. The optimal cut-off value of 1.22 for AGR was used to divide patients into two groups ( $\leq 1.22, n=71 ;>1.22, n=344)$. Optimal cut-off values for neutrophil to lymphocyte ratio (NLR), monocyte to lymphocyte ratio (MLR), and platelet to lymphocyte ratio (PLR) were 2.17, 0.30, and 179.83, respectively. Lower limits of the normal range, which were $130 \mathrm{~g} / \mathrm{L}$ for males and $115 \mathrm{~g} / \mathrm{L}$ for females, were used as cut-off values for hemoglobin $(\mathrm{Hb})$. The upper limit of the normal range, $125 \mathrm{U} / \mathrm{L}$, was used as the cut-off value for alkaline phosphatase (ALP), while 1.5 times the upper limit of $245 \mathrm{U} / \mathrm{L}$ was used for lactate dehydrogenase (LDH).

\section{Associations between AGR and primary cohort patient clinicopathological characteristics}

Associations between AGR and clinicopathological characteristics in the 416 primary cohort patients are shown in Table 2. Low AGR was associated with older age at surgery $(P<0.001)$, higher T stage $(P<0.001), N$ stage $(P=0.005)$, and Fuhrman grade $(P=0.001)$, larger tumor size $(P<0.001)$, the presence of tumor necrosis $(P<0.001)$ and lymphovascular invasion $(P=0.001)$, lower $\mathrm{Hb}$ concentration $(P<0.001)$, higher AKP $(P<0.001)$ and LDH $(P=0.005)$ concentrations, and lower BMI $(P<0.001)$.

\section{Prognostic value of AGR}

OS $(P<0.001)$ and CSS $(P<0.001)$ were worse in patients with low AGRs $(\leq 1.22)$ than in patients with high AGRs ( $>1.22)$ prior to propensity score matching (PSM) (Figure 1A, 1B). Because some clinicopathological characteristics differed between the low and high AGR patient groups (Table 2), we performed PSM to minimize these differences. In the PSM analysis, 52 patients selected from the high AGR group were each paired with one low AGR patient using a nearest-neighbor algorithm. After PSM, clinicopathological characteristics were balanced and evenly distributed between the low and high AGR groups (all $P>0.05$ ) (Table 2). Kaplan-Meier survival curves for the post-PSM low and high AGR groups confirmed that OS and CSS were still worse in low AGR patients, even when controlling for differences in clinicopathological characteristics (Figure 1C, 1D).

The results of the multivariate analysis for OS and CSS before PSM in the primary cohort are shown in Table 3. Because AGR, NLR, MLR, PLR, GPS, and mGPS were correlated initially, we then conducted multivariate analysis for these inflammation-based prognostic scores. AGR (HR: 6.529; 95\% CI: 3.036-14.042; $P<0.001$ ), NLR $(P=0.001), \operatorname{MLR}(P=0.001), \operatorname{GPS}(P=0.001)$, mGPS $(P=0.026)$, T stage $(P=0.005)$, and $\mathrm{N}$ stage $(P=0.041)$ were independently associated with OS. However, PLR $(P=0.909)$ was not an independent prognostic factor. The results of the multivariate analysis for CSS were very similar to those for OS.

\section{Comparison of the discriminatory abilities of AGR and established inflammation-based prognostic scores}

To assess the discriminatory ability of AGR compared to that of the established inflammation-based prognostic scores, we generated ROC curves for OS at the 3- and 5-year follow-up examinations, (Figure 2). Table 4 shows a comparison of the discriminatory ability of AGR to that of the other inflammation-based prognostic scores. To ensure that comparisons were rational, continuous indices were compared to continuous AGR data, while categorical indices were compared to the high and low AGR categories. While the area under the curve (AUC) value tended to be higher for AGR than for the other inflammation-based prognostic scores at both 3 years and 5 years, this difference was only significant for some of the prognostic scores (Table 3). 


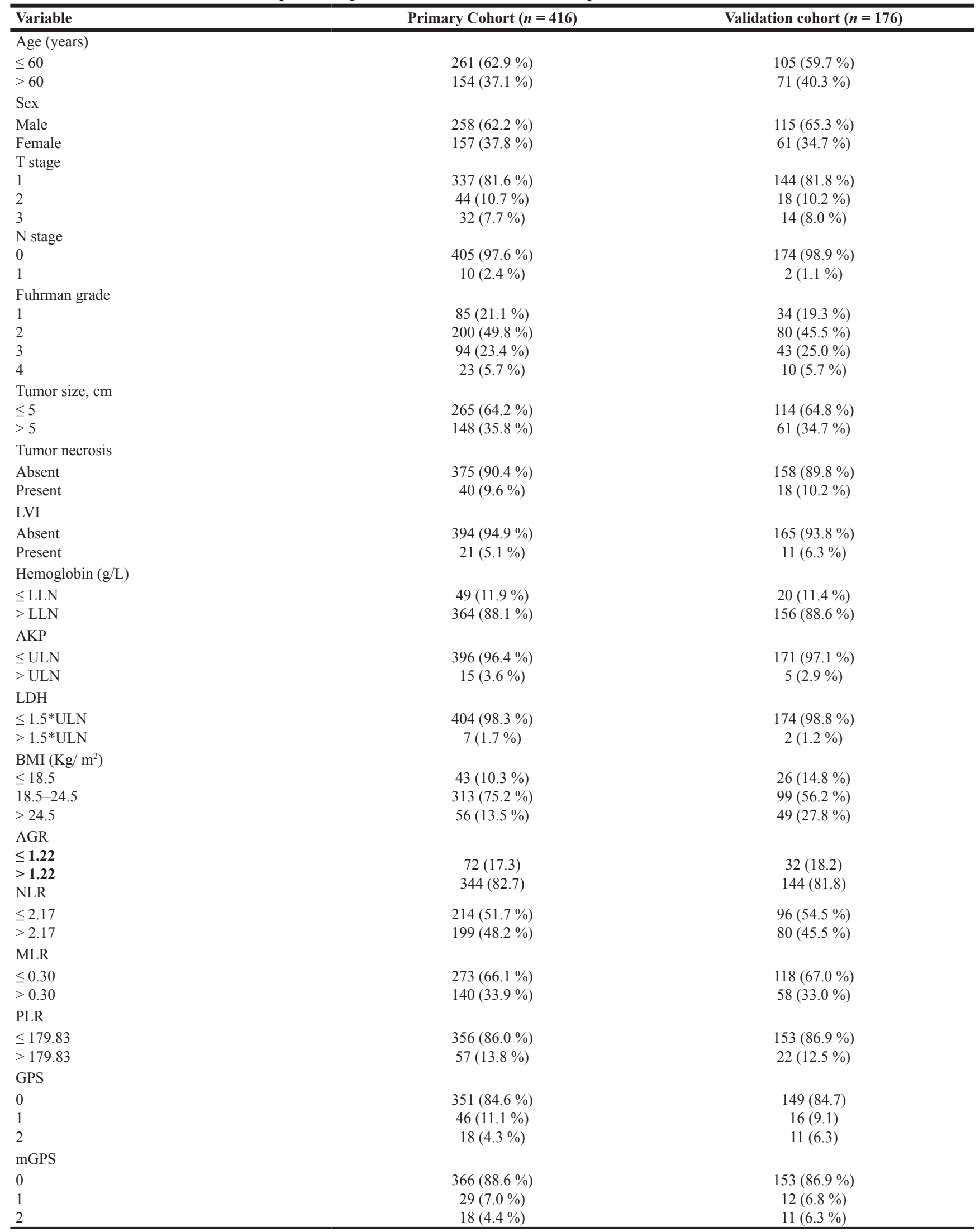

Abbreviations: PSM, propensity score matching; LVI, lymphovascular invasion; AKP, alkaline phosphatase; LDH, lactate dehydrogenase; BMI, body mass index; AGR, albumin to globulin ratio; NLR, neutrophil to lymphocyte ratio; MLR, monocyte to lymphocyte ratio; PLR, the platelet to lymphocyte ratio; GPS, Glasgow Prognostic Score; mGPS, modified Glasgow Prognostic Score. 
Table 2: Associations between AGR and clinicopathological characteristics in primary cohort patients before and after PSM

\begin{tabular}{|c|c|c|c|c|c|c|}
\hline \multirow[b]{2}{*}{ Variable } & \multicolumn{3}{|c|}{ Pre-PSM } & \multicolumn{3}{|c|}{ Post-PSM } \\
\hline & $\begin{array}{c}\mathbf{A G R} \leq 1.22 \\
(n=72)\end{array}$ & $\begin{array}{c}\text { AGR }>1.22 \\
(n=344)\end{array}$ & $P$ value & $\begin{array}{c}\mathrm{AGR} \leq 1.22 \\
(n=52)\end{array}$ & $\begin{array}{c}\text { AGR }>1.22 \\
(n=52)\end{array}$ & $P$ value \\
\hline Age (years) & & & $<0.001 *$ & & & 0.695 \\
\hline $\begin{array}{l}\leq 60 \\
>60\end{array}$ & $\begin{array}{l}29(40.8 \%) \\
42(59.2 \%)\end{array}$ & $\begin{array}{l}232(67.4 \%) \\
112(32.6 \%)\end{array}$ & & $\begin{array}{l}24(46.2 \%) \\
28(53.8 \%)\end{array}$ & $\begin{array}{l}26(50.0 \%) \\
26(50.0 \%)\end{array}$ & \\
\hline Sex & & & 0.399 & & & 0.543 \\
\hline $\begin{array}{l}\text { Male } \\
\text { Female } \\
\end{array}$ & $\begin{array}{l}41(57.7 \%) \\
30(42.3 \%)\end{array}$ & $\begin{array}{l}217(63.1 \%) \\
127(36.9 \%)\end{array}$ & $<0001 *$ & $\begin{array}{l}31(59.6 \%) \\
21(40.4 \%)\end{array}$ & $\begin{array}{l}34(65.4 \%) \\
18(34.3 \%)\end{array}$ & \\
\hline $\begin{array}{l}1 \\
2 \\
3\end{array}$ & $\begin{array}{l}42(59.2 \%) \\
12(16.9 \%) \\
17(23.9 \%)\end{array}$ & $\begin{array}{c}295(86.3 \%) \\
32(9.4 \%) \\
15(4.4 \%)\end{array}$ & & $\begin{array}{c}40(76.9 \%) \\
8(15.4 \%) \\
4(7.7 \%)\end{array}$ & $\begin{array}{l}37(71.1 \%) \\
7(13.5 \%) \\
8(15.4 \%)\end{array}$ & 0.468 \\
\hline $\begin{array}{c}\text { N stage } \\
0 \\
1\end{array}$ & $\begin{array}{c}66(93.0 \%) \\
5(7.0 \%)\end{array}$ & $\begin{array}{c}339(98.5 \%) \\
5(1.5 \%)\end{array}$ & $0.005^{*}$ & $\begin{array}{c}50(96.2 \%) \\
2(3.8 \%)\end{array}$ & $\begin{array}{c}50(96.2 \%) \\
2(3.8 \%)\end{array}$ & 1 \\
\hline $\begin{array}{l}\text { Fuhrman grade } \\
\qquad \begin{array}{c}1 \\
2 \\
3 \\
4\end{array}\end{array}$ & $\begin{array}{c}8(11.8 \%) \\
30(44.1 \%) \\
20(29.4 \%) \\
10(14.7 \%)\end{array}$ & $\begin{array}{c}77(23.1 \%) \\
170(50.9 \%) \\
74(22.2 \%) \\
13(3.9 \%)\end{array}$ & $0.001 *$ & $\begin{array}{c}6(13.3 \%) \\
25(55.6 \%) \\
11(24.4 \%) \\
3(6.7 \%)\end{array}$ & $\begin{array}{c}1(2.2 \%) \\
25(55.6 \%) \\
15(33.3 \%) \\
4(8.9 \%)\end{array}$ & 0.228 \\
\hline $\begin{array}{l}\text { Tumor size, cm } \\
\quad \leq 5 \\
\quad>5\end{array}$ & $\begin{array}{l}30(42.9 \%) \\
40(57.1 \%)\end{array}$ & $\begin{array}{l}235(68.5 \%) \\
108(31.5 \%)\end{array}$ & $<0.001^{*}$ & $\begin{array}{l}28(53.8 \%) \\
24(46.2 \%)\end{array}$ & $\begin{array}{l}31(59.6 \%) \\
21(40.4 \%)\end{array}$ & 0.553 \\
\hline $\begin{array}{c}\text { Tumor necrosis } \\
\text { Absent } \\
\text { Present }\end{array}$ & $\begin{array}{l}56(78.9 \%) \\
15(21.1 \%)\end{array}$ & $\begin{array}{c}319(92.7 \%) \\
25(7.3 \%)\end{array}$ & $<0.001 *$ & $\begin{array}{c}45(86.5 \%) \\
7(13.5 \%)\end{array}$ & $\begin{array}{l}42(80.8 \%) \\
10(19.2 \%)\end{array}$ & 0.426 \\
\hline LVI & & & $0.001 *$ & & & 0.462 \\
\hline $\begin{array}{l}\text { Absent } \\
\text { Present }\end{array}$ & $\begin{array}{c}62(87.3 \%) \\
9(12.7 \%)\end{array}$ & $\begin{array}{c}332(96.5 \%) \\
12(3.5 \%)\end{array}$ & & $\begin{array}{c}49(94.2 \%) \\
3(5.8 \%)\end{array}$ & $\begin{array}{c}47(90.4 \%) \\
5(9.6 \%)\end{array}$ & \\
\hline Hemoglobin (g/L) & & & $<0.001 *$ & & & 0.631 \\
\hline $\begin{array}{l}\leq \mathrm{LLN} \\
>\mathrm{LLN}\end{array}$ & $\begin{array}{l}27(38.6 \%) \\
43(61.4 \%)\end{array}$ & $\begin{array}{c}22(6.4 \%) \\
321(93.6 \%)\end{array}$ & & $\begin{array}{l}12(23.1 \%) \\
40(76.9 \%)\end{array}$ & $\begin{array}{l}10(19.2 \%) \\
42(80.8 \%)\end{array}$ & \\
\hline AKP & & & $<0.001 *$ & & & 1 \\
\hline $\begin{array}{l}\leq \text { ULN } \\
>\text { ULN }\end{array}$ & $\begin{array}{c}62(87.3 \%) \\
9(12.7 \%)\end{array}$ & $\begin{array}{c}334(98.2 \%) \\
6(1.8 \%)\end{array}$ & & $\begin{array}{c}48(92.3 \%) \\
4(7.7 \%)\end{array}$ & $\begin{array}{c}48(92.3 \%) \\
4(7.7 \%)\end{array}$ & \\
\hline $\begin{aligned} & \mathrm{LDH} \\
\leq & 1.5^{*} \mathrm{ULN} \\
> & 1.5^{*} \mathrm{ULN}\end{aligned}$ & $\begin{array}{c}67(94.4 \%) \\
4(5.6 \%)\end{array}$ & $\begin{array}{c}337(99.1 \%) \\
3(0.9 \%)\end{array}$ & $0.005^{*}$ & $\begin{array}{c}52(100.0 \%) \\
0(0.0 \%)\end{array}$ & $\begin{array}{c}51(98.1 \%) \\
1(1.9 \%)\end{array}$ & 0.315 \\
\hline $\begin{array}{c}\mathrm{BMI}\left(\mathrm{Kg} / \mathrm{m}^{2}\right) \\
\leq 18.5 \\
18.5-24.5 \\
>24.5\end{array}$ & $\begin{array}{l}28(38.9 \%) \\
29(40.3 \%) \\
14(19.4 \%)\end{array}$ & $\begin{array}{c}15(4.4 \%) \\
284(82.5 \%) \\
42(12.2 \%)\end{array}$ & $<0.001^{*}$ & $\begin{array}{l}22(42.3 \%) \\
20(38.5 \%) \\
10(19.2 \%)\end{array}$ & $\begin{array}{l}13(25.0 \%) \\
29(55.8 \%) \\
10(19.2 \%)\end{array}$ & 0.138 \\
\hline
\end{tabular}

${ }^{*} p<0.05$

Abbreviations: PSM, propensity score matching; LLN, lower limit of normal; ULN, upper limit of normal; LVI, lymphovascular invasion; AKP, alkaline phosphatase; LDH, lactate dehydrogenase; BMI, body mass index; AGR, albumin to globulin ratio. 


\section{Predictive nomogram for OS}

Multivariate analysis indicated that $\mathrm{T}$ stage, $\mathrm{N}$ stage, AGR, NLR, MLR, GPS, and mGPS were independent risk factors for OS (Table 3); these scores became candidates for inclusion in the final model. Ultimately, the optimal nomogram integrated $\mathrm{T}$ stage, AGR, NLR, and MLR to predict 3- and 5-year OS (Figure 3A). The initial concordance index (C-index) values of the Leibovich score [13], SSIGN score [14] and TNM stage of $0.847,0.838$, and 0.806 , respectively, increased to $0.899,0.898$, and 0.884 when the AGR (C-index 0.783) was incorporated. The C-index for the nomogram was 0.914, indicating that it had a better predictive ability than Leibovich score $(P<0.001)$, SSIGN score $(P<0.001)$, and TNM stage $(P<0.001)$. Calibration plots for the probability

A

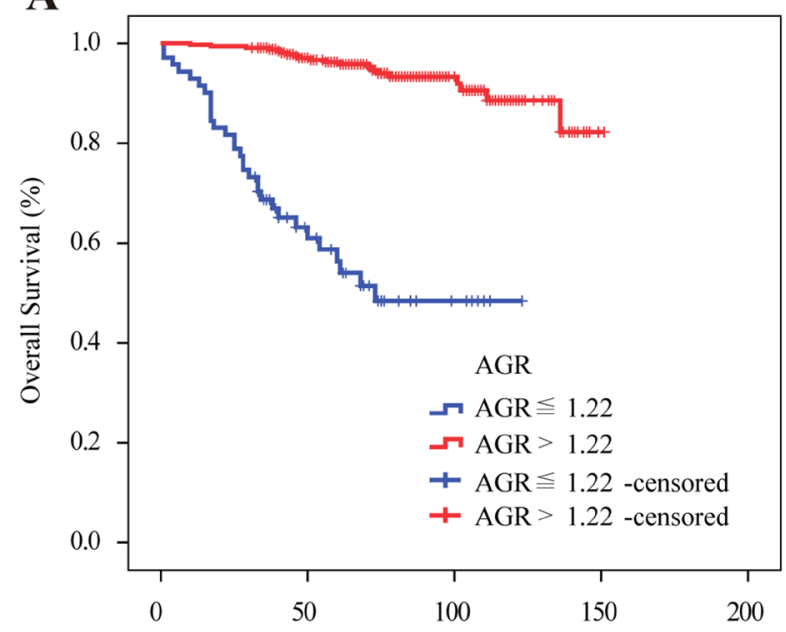

C

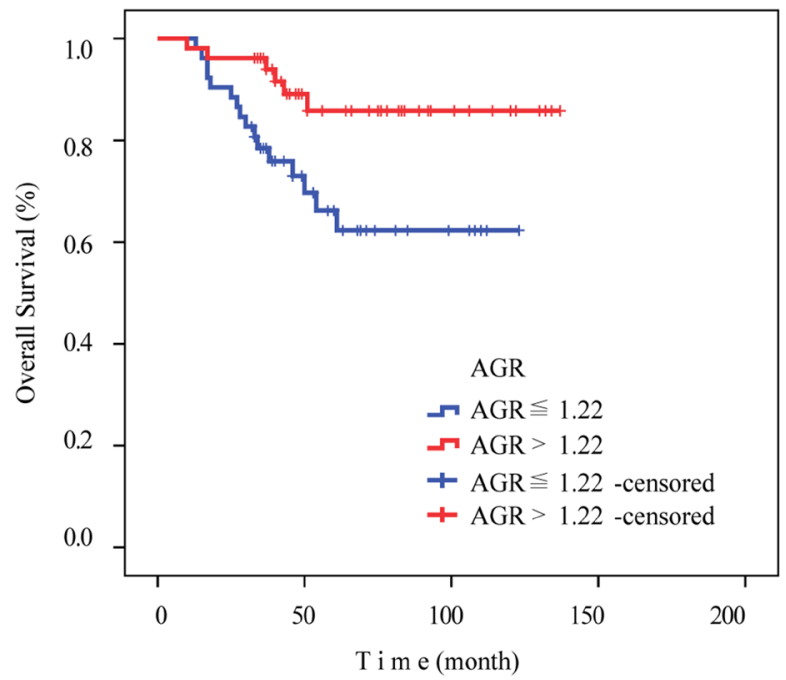

of survival at 3 or 5 years after surgery demonstrated virtually no departures from ideal predictions, confirming the internal validity of the results (Figure 3B, 3C).

\section{Validation of predictive accuracy of the nomogram for $\mathrm{OS}$}

Calibration curves revealed that predictions of 3- and 5-year survival probability obtained using the nomogram were highly consistent with the actual patient OS values (Figure 3D, 3E). The C-index for OS predictions generated using the nomogram was 0.895 in the validation cohort; this was again higher than the $\mathrm{C}$-index values of the Leibovich score $(0.808, P<0.001)$, SSIGN score $(0.819, P<0.001)$, and TNM stage $(0.771$, $P<0.001)$.
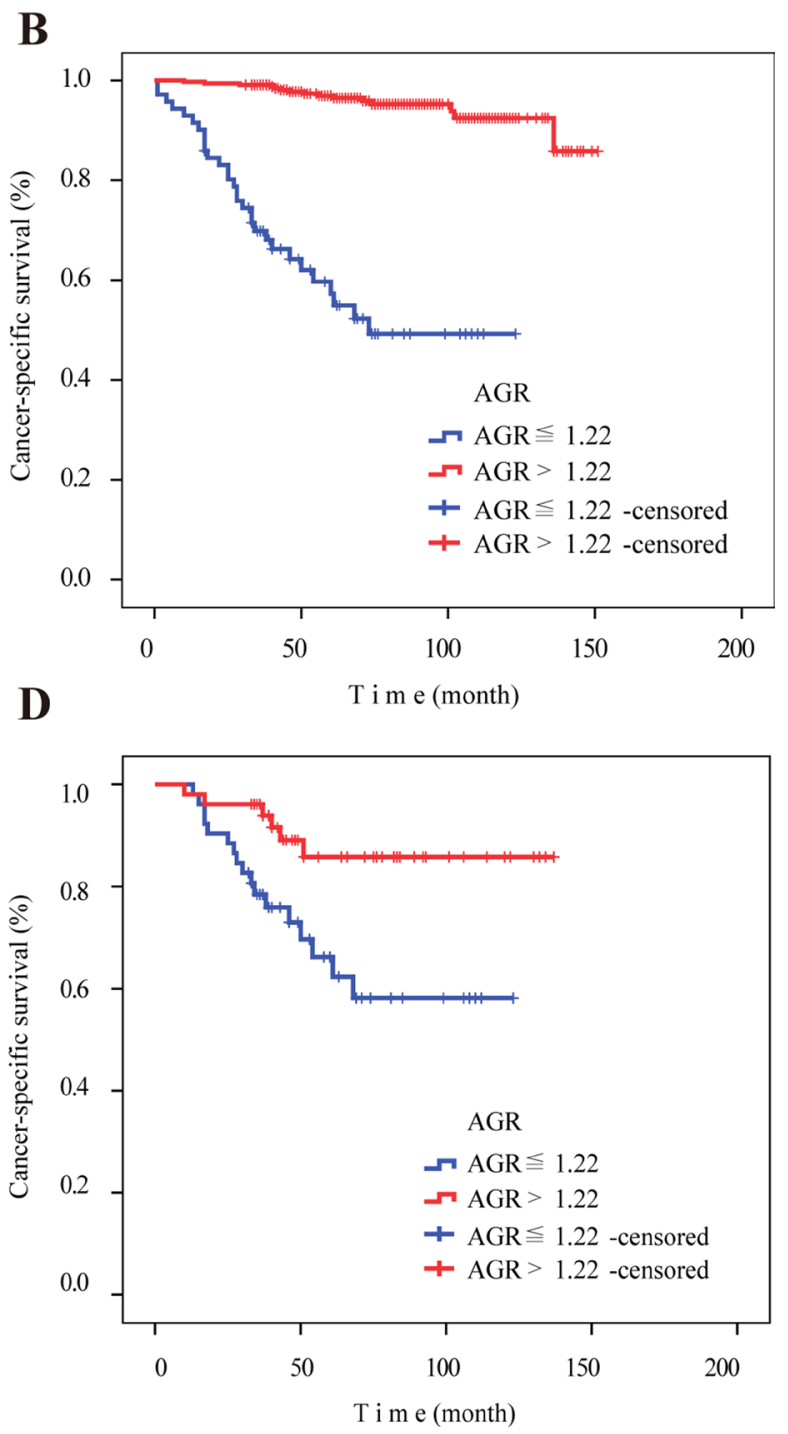

Figure 1: Kaplan-Meier curves for overall survival and cancer-specific survival according to preoperative AGR before and after PSM. (A) Overall survival $(P<0.001)$ and $(\mathbf{B})$ cancer-specific survival $(P<0.001)$ were worse in patients with low AGR $(\leq 1.22)$ before PSM. $(\mathbf{C})$ Overall survival $(P=0.012)$ and $(\mathbf{D})$ cancer-specific survival $(P=0.007)$ were worse in patients with low AGR $(\leq 1.22)$ after PSM. Abbreviation: AGR, albumin to globulin ratio.PSM, propensity score matching. 
Table 3: Overall survival, cancer-specific survival, and multivariate analyses in primary cohort patients before PSM

\begin{tabular}{|c|c|c|c|c|}
\hline \multirow{2}{*}{ Variable } & \multicolumn{2}{|c|}{ Multivariate analysis for OS } & \multicolumn{2}{|c|}{ Multivariate analysis for CSS } \\
\hline & HR (95 \% CI) & $p$ value & HR (95\% CI) & $p$ value \\
\hline Age(years) & & $0.323^{\mathrm{a}}$ & & $0.139^{\mathrm{a}}$ \\
\hline$>60$ years vs. $\leq 60$ years & $1.438(0.700-2.957)$ & & $1.804(0.825-3.941)$ & \\
\hline \multicolumn{5}{|l|}{ Sex } \\
\hline \multicolumn{5}{|l|}{ Male vs. Female } \\
\hline T stage & & $0.005^{* a}$ & & $0.003^{* a}$ \\
\hline 1 & reference & & reference & \\
\hline 2 & $2.188(0.874-5.480)$ & $0.094^{\mathrm{a}}$ & $2.548(0.950-6.834)$ & $0.063^{\mathrm{a}}$ \\
\hline 3 & $5.972(2.049-17.407)$ & $0.001^{* a}$ & $6.950(2.298-21.021))$ & $0.001^{* a}$ \\
\hline $\mathrm{N}$ stage & & $0.041^{* a}$ & & $0.024^{*}$ a \\
\hline 1 vs. 0 & $3.374(1.050-10.846)$ & & $3.985(1.203-13.024)$ & \\
\hline Fuhrman grade & & $0.156^{\mathrm{a}}$ & & $0.140^{\mathrm{a}}$ \\
\hline 1 & reference & & reference & \\
\hline 2 & $2.037(0.569-7.300)$ & $0.274^{\mathrm{a}}$ & $1.441(0.384-5.401)$ & $0.588^{\mathrm{a}}$ \\
\hline 3 & $2.119(0.572-7.855)$ & $0.261^{\mathrm{a}}$ & $1.900(0.498-7.250)$ & $0.347^{\text {a }}$ \\
\hline 4 & $0.617(0.119-3.210)$ & $0.566^{\mathrm{a}}$ & $0.413(0.075-2.294)$ & $0.312^{\mathrm{a}}$ \\
\hline $\begin{array}{l}\text { Tumor size, cm } \\
>5 \text { vs. } \leq 5\end{array}$ & $1.601(0.703-3.646)$ & $0.263^{\mathrm{a}}$ & $1.296(0.538-3.120)$ & $0.564^{\mathrm{a}}$ \\
\hline Tumor necrosis & & $0.056^{* a}$ & & $0.079^{\mathrm{a}}$ \\
\hline Present vs. Absent & $2.038(0.982-4.226)$ & & $2.019(0.922-4.423)$ & \\
\hline LVI & & $0.127^{\mathrm{a}}$ & & $0.143^{\text {a }}$ \\
\hline Present vs. Absent & $2.298(0.790-6.682)$ & & $2.278(0.757-6.856)$ & \\
\hline Hemoglobin $(\mathrm{g} / \mathrm{L})$ & & $0.829^{\mathrm{a}}$ & & $0.674^{\mathrm{a}}$ \\
\hline$\leq \mathrm{LLN}$ vs. $>$ LLN & $1.096(0.476-2.528)$ & & $0.824(0.336-2.025)$ & \\
\hline AKP & & $0.507^{\mathrm{a}}$ & & $0.709^{\mathrm{a}}$ \\
\hline$>\mathrm{ULN}$ vs. $\leq \mathrm{ULN}$ & $0.652(0.184-2.306)$ & & $0.778(0.208-2.911)$ & \\
\hline LDH & & $0.824^{\mathrm{a}}$ & & $0.611^{\mathrm{a}}$ \\
\hline$>1.5^{*} \mathrm{ULN}$ vs. $\leq 1.5 * \mathrm{ULN}$ & $1.198(0.244-5.887)$ & & $1.520(0.303-7.624)$ & \\
\hline AGR & & $<0.0^{01^{*} \mathrm{a}}$ & & $<0.001^{*_{a}}$ \\
\hline$\leq 1.22$ vs. $>1.22$ & $6.529(3.036-14.042)$ & & $8.806(3.891-19.928)$ & \\
\hline NLR & & $0.001^{* \mathrm{~b}}$ & & $0.001^{* b}$ \\
\hline$>2.17$ vs. $\leq 2.17$ & $3.689(1.752-7.766)$ & & $4.076(1.805-9.203)$ & \\
\hline MLR & & $0.001^{* \mathrm{~b}}$ & & $0.004^{*} \mathrm{~b}$ \\
\hline$>0.30$ vs. $\leq 0.30$ & $3.406(1.670-6.946)$ & & $2.961(1.416-6.190)$ & \\
\hline PLR & & $0.909^{\mathrm{b}}$ & & $0.828^{\mathrm{b}}$ \\
\hline$>179.83$ vs. $\leq 179.83$ & $1.053(0.435-2.545)$ & & $1.108(0.439-2.795)$ & \\
\hline GPS & & $0.001^{* \mathrm{~b}}$ & & $0.003^{* b}$ \\
\hline 0 & Reference & & Reference & \\
\hline 1 & $4.167(2.000-8.684)$ & $<0.001^{* b}$ & $3.938(1.799-8.618)$ & $0.001^{* b}$ \\
\hline 2 & $1.287(0.372-4.455)$ & $0.690 \mathrm{~b}$ & $1.579(0.444-5.618)$ & $0.481^{\mathrm{b}}$ \\
\hline mGPS & & $0.026^{* \mathrm{~b}}$ & & $0.158^{\mathrm{b}}$ \\
\hline 0 & reference & & reference & \\
\hline 1 & $3.156(1.348-7.390)$ & $0.008^{* \mathrm{~b}}$ & $2.537(0.980-6.571)$ & $0.055^{\mathrm{b}}$ \\
\hline 2 & $0.955(0.286-3.189)$ & $0.940^{\mathrm{b}}$ & $1.164(0.339-3.999)$ & $0.809^{\mathrm{b}}$ \\
\hline
\end{tabular}

$* p<0.05$

Abbreviations: PSM, propensity score matching; OS, overall survival; CSS, cancer-specific survival; LLN, lower limit of normal; ULN, upper limit of normal; AGR, albumin to globulin ratio; LVI, lymphovascular invasion; AKP, alkaline phosphatase; LDH, lactate dehydrogenase; NLR, neutrophil to lymphocyte ratio; MLR, monocyte to lymphocyte ratio; PLR, platelet to lymphocyte ratio; GPS, Glasgow Prognostic Score; mGPS, modified Glasgow Prognostic Score. ${ }^{a}$ Age, T stage, $\mathrm{N}$ stage, Fuhrman grade, tumor size, tumor necrosis, LVI, hemoglobin, AKP, LDH, and AGR variables were tested in a multivariate analysis. ${ }^{\mathrm{b}}$ Established inflammation-based prognostic scores were evaluated together with age, T stage, N stage, Fuhrman grade, tumor size, tumor necrosis, LVI, hemoglobin, AKP, and LDH variables; multivariate analysis for these inflammation-based prognostic scores was then conducted. 


\section{DISCUSSION}

In this study, we analyzed the prognostic power of AGR and the ability of a nomogram that combined AGR with other inflammation-based prognostic scores and clinicopathological characteristics to predict 3- and 5-year survival in patients with localized or locally advanced CCRCC. We found that AGR was an independent prognostic factor for OS and CSS and had a better discriminatory ability than the established inflammationbased prognostic scores. Furthermore, the nomogram that integrated AGR with T stage, NLR, and MLR predicted OS with an accuracy of 0.914 .

In recent years, the relationship between AGR, which is a classic indicator of liver function, and cancer has received increasing attention. Research has shown that low AGR is correlated with tumor incidence and mortality rates among healthy people [15], and that AGR is associated with prognosis in breast [9], colorectal [10], nasopharyngeal [11] and lung cancer patients [12]. To our knowledge, the present study is the first to analyze the association between AGR and survival in CCRCC patients.

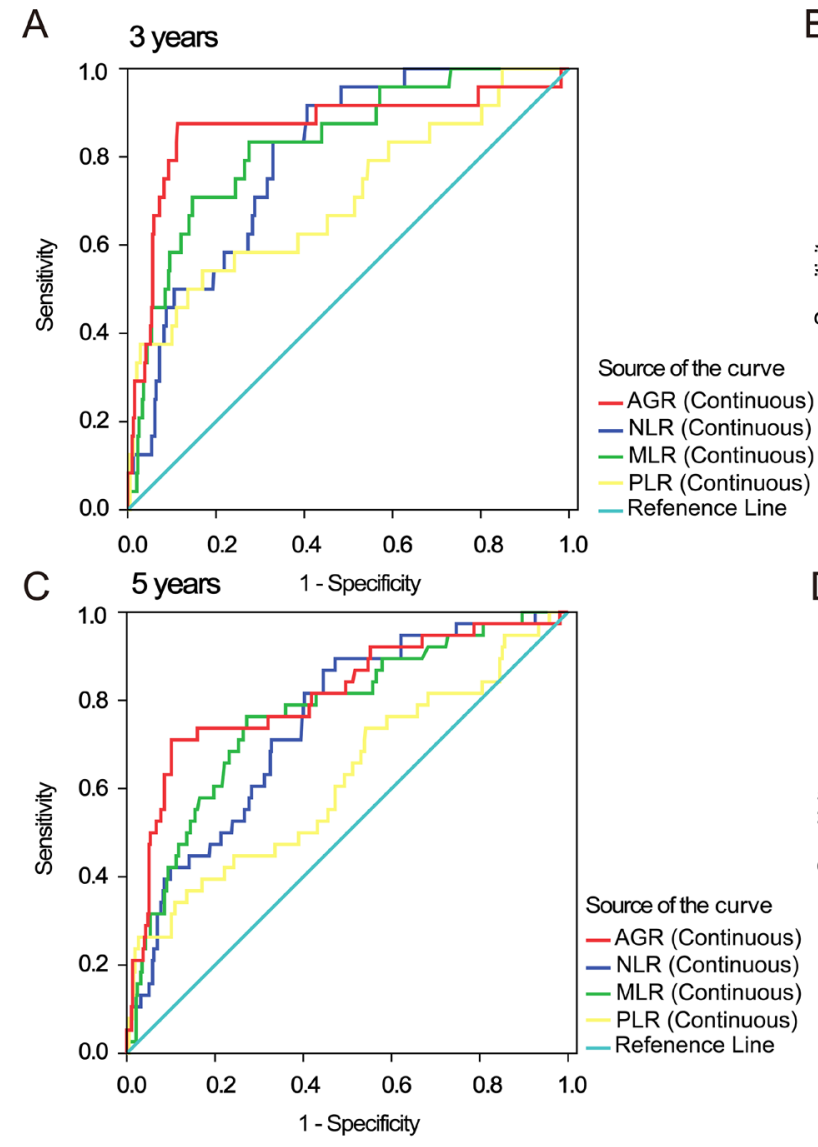

Several potential mechanisms involved in nutrition and inflammation might contribute to the prognostic value of AGR in cancer. Malnutrition is relatively common in cancer patients, and it often develops into cancer cachexia, which accelerates disease progression. Inflammation associated with tumors involves not only inflammatory factors generated by tumor cells, but also those released during the processes of tissue remodeling and rehabilitation and angiogenesis [16]. Consequently, in addition to changes in the local tumor microenvironment, changes in levels of peripheral inflammatory factors, such as tumor necrosis factor (TNF), interleukin (IL)-1, -6, and -8 , and vascular endothelial growth factor (VEGF), also promote tumor growth and metastasis [16-18]. Thus, malignant tumor progression promotes malnutrition and increases inflammation, and vice versa. Effective, practical indicators of nutritional and inflammation status may therefore be useful not only for quantifying tumor malignancy, but also for evaluating patient prognosis. AGR may be a particularly useful indicator in this regard, as it combines nutritional and inflammatory indices. Generally, serum albumin level is thought to reflect the body's nutritional status, and recent studies have shown

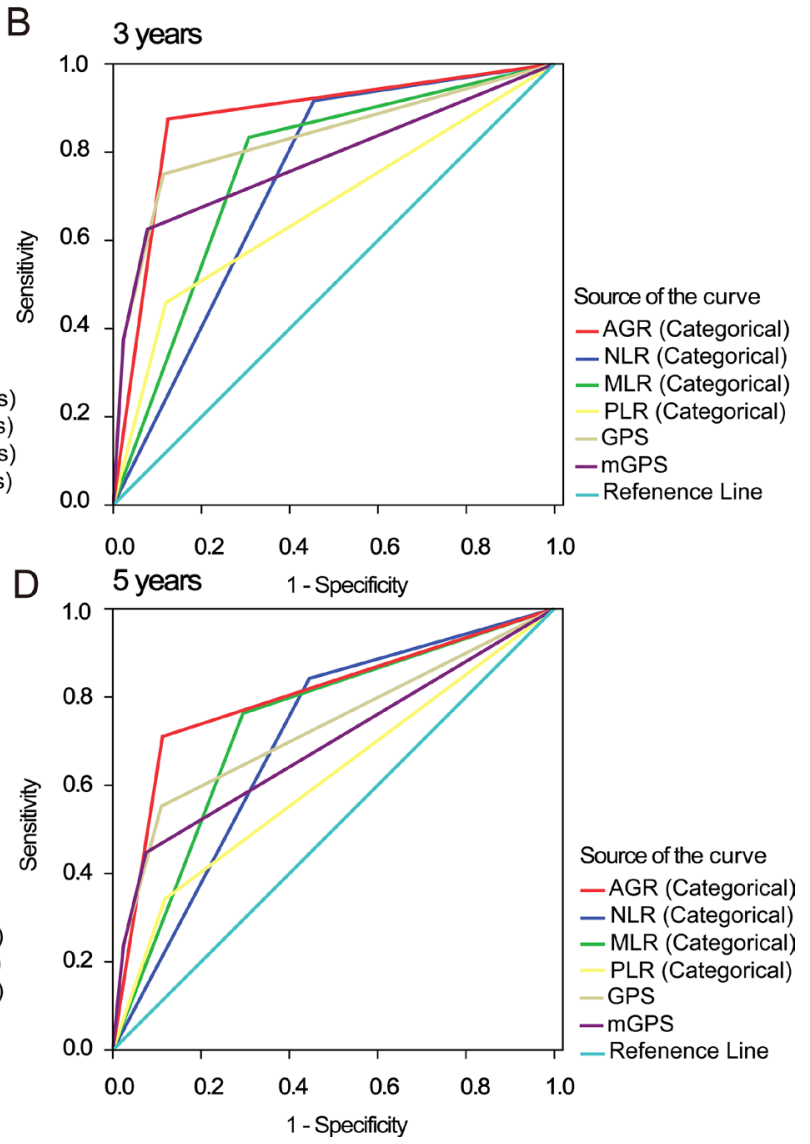

Figure 2: ROC curves for inflammation-based 3- and 5-year prognostic scores. ROC curves for AGR, NLR, MLR, PLR (continuous and categorical), GPS, and mGPS for 3- and 5-year survival. Abbreviations: AGR, albumin to globulin ratio; NLR, neutrophil to lymphocyte ratio; MLR, monocyte to lymphocyte ratio; PLR, platelet to lymphocyte ratio; GPS, Glasgow Prognostic Score; mGPS, modified Glasgow Prognostic Score. 
Table 4: Comparison of the discriminatory ability of inflammation-based prognostic scores in primary cohort patients before PSM

\begin{tabular}{|c|c|c|c|c|}
\hline Period & $\mathbf{A U C}$ & $95 \% \mathrm{CI}$ & $p$ value & $p$ value for comparison to AGR $\S$ \\
\hline \multicolumn{5}{|l|}{ 3-year (continuous) } \\
\hline AGR (continuous) & 0.866 & $0.768-0.965$ & $<0.001 *$ & - \\
\hline NLR (continuous) & 0.799 & $0.727-0.871$ & $<0.001 *$ & 0.246 \\
\hline MLR (continuous) & 0.828 & $0.745-0.911$ & $<0.001 *$ & 0.458 \\
\hline PLR (continuous) & 0.705 & $0.584-0.826$ & $0.001 *$ & $0.028 *$ \\
\hline \multicolumn{5}{|l|}{ 3-year (categorical) } \\
\hline AGR (categorical) & 0.875 & $0.797-0.954$ & $<0.001 *$ & - \\
\hline NLR (categorical) & 0.731 & $0.648-0.814$ & $<0.001 *$ & $0.004 *$ \\
\hline MLR (categorical) & 0.763 & $0.671-0.855$ & $<0.001 *$ & $0.024 *$ \\
\hline PLR (categorical) & 0.670 & $0.543-0.797$ & $0.005 *$ & $<0.001 *$ \\
\hline GPS & 0.831 & $0.726-0.936$ & $<0.001 *$ & 0.239 \\
\hline mGPS & 0.781 & $0.661-0.901$ & $<0.001 *$ & $0.047 *$ \\
\hline 5-year (continuous) & . & & & \\
\hline AGR (continuous) & 0.812 & $0.730-0.893$ & $<0.001 *$ & - \\
\hline NLR (continuous) & 0.747 & $0.672-0.823$ & $<0.001 *$ & 0.224 \\
\hline MLR (continuous) & 0.768 & $0.688-0.849$ & $<0.001 *$ & 0.371 \\
\hline PLR (continuous) & 0.621 & $0.518-0.724$ & $0.014^{*}$ & $0.003 *$ \\
\hline \multicolumn{5}{|l|}{ 5-year (categorical) } \\
\hline AGR (categorical) & 0.799 & $0.712-0.886$ & $<0.001 *$ & - \\
\hline NLR (categorical) & 0.699 & $0.620-0.777$ & $<0.001 *$ & 0.060 \\
\hline MLR (categorical) & 0.734 & $0.651-0.817$ & $<0.001 *$ & 0.186 \\
\hline PLR (categorical) & 0.612 & $0.509-0.715$ & $0.023 *$ & $<0.001 *$ \\
\hline GPS & 0.728 & $0.628-0.828$ & $<0.001 *$ & 0.090 \\
\hline mGPS & 0.690 & $0.585-0.794$ & $<0.001 *$ & $0.010^{*}$ \\
\hline
\end{tabular}

$* p<0.05$.

$\S A U C$ values for AGR and for other inflammation-based prognostic scores were compared using MedCalc software. Continuous indexes were compared with continuous AGR scores, while categorical indexes were compared with AGR score categories.

Abbreviations: PSM, propensity score matching; AUC, area under the curves; NLR, neutrophil to lymphocyte ratio; MLR, monocyte to lymphocyte ratio; PLR, platelet to lymphocyte ratio; GPS, Glasgow Prognostic Score; mGPS, modified Glasgow Prognostic Score.

that it is also an effective indicator of inflammatory state $[6,19,20]$. Serum globulin plays an important role in the body's immune and inflammatory responses as well. High serum globulin levels result from the accumulation of acute-phase proteins and immunoglobulins, and increased expression of these proteins is indicative of a persistent inflammatory state [10]. By combining the albumin and globulin indicators in a single measurement, AGR more accurately reflects the body's nutritional and inflammatory states and may be particularly useful as an outcome indicator for cancer patients.

Previous studies have demonstrated that established inflammation-based prognostic scores, such as NLR, MLR, PLR, GPS and mGPS, are effective in assessing the prognosis of RCC patients [21-25]. Here, we compared the prognostic ability of AGR to that of other established inflammation-based prognostic scores in RCC patients. Generally, as shown in Table 3, the prognostic ability of AGR was superior to that of the established inflammationbased prognostic scores.
Usually, outcome predictions for RCC patients are based on clinicopathological characteristics, such as the TNM staging system and the Fuhrman grading system, and few existing models can successfully predict survival of patients with localized or locally advanced CCRCC. Here, we identified a nomogram that incorporated inflammationbased prognostic scores and traditional staging systems to predict 3-and 5-year OS in these patients more accurately than Leibovich score, SSIGN score, and TNM stage. The nomogram was equally effective in predicting outcomes in the validation cohort patients, and calibration plots for both the primary and validation cohorts revealed that the predicted survival probabilities were closely correlated with the actual survival rates. The well-established Leibovich and SSIGN scores incorporate multiple clinical and pathologic variables, including $\mathrm{T}$ stage, $\mathrm{N}$ stage, $\mathrm{M}$ stage, tumor size, Fuhrman grade, and necrosis. Here, we evaluated the prognostic value of all of these variables individually and found that tumor size, Fuhrman grade, and necrosis were not independent prognostic factors 
in our patient cohort, suggesting that the two classical prognostic scores may not be useful for patients with localized or locally advanced CCRCC. The incorporation of inflammation-based prognostic scores in addition to clinicopathological characteristics in our nomogram likely enhanced its predictive accuracy. Moreover, AGR,

A

Points

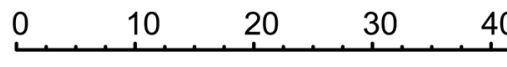

T stage

AGR

T2

NLR

MLR

T1 $\leq 1.22$

$>2.17$

Total Points

$>2.17>0.30$

3-year survival

5-year survival

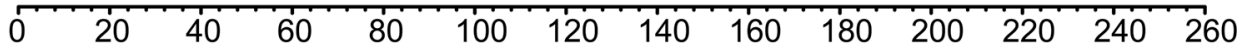

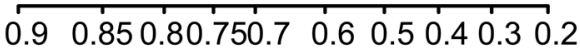

\section{$\begin{array}{llllllll}0.9 & 0.850 .80 .750 .7 & 0.6 & 0.5 & 0.4 & 0.3 & 0.2 & 0.1\end{array}$}

B

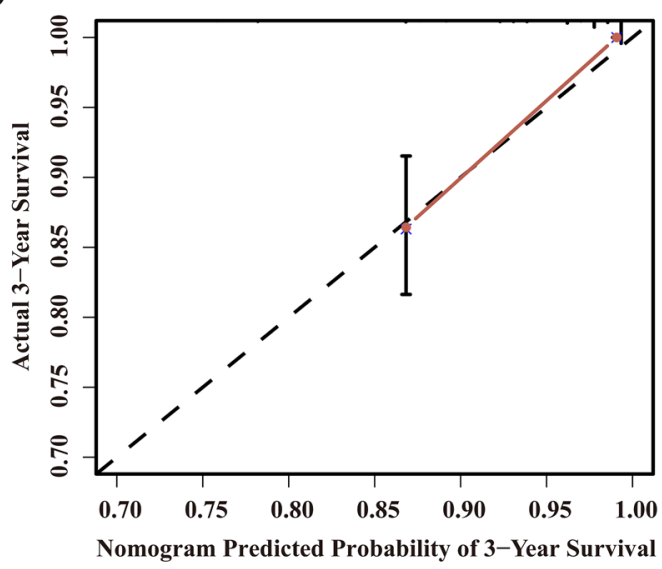

$\mathrm{D}$

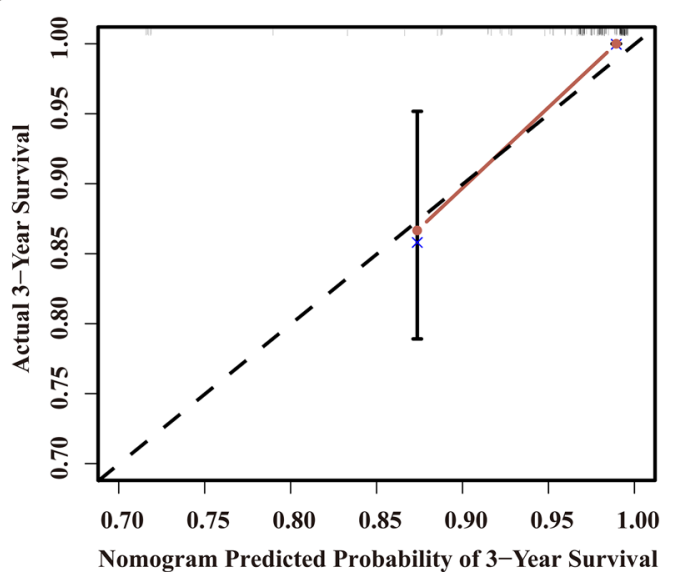

$\mathrm{C}$

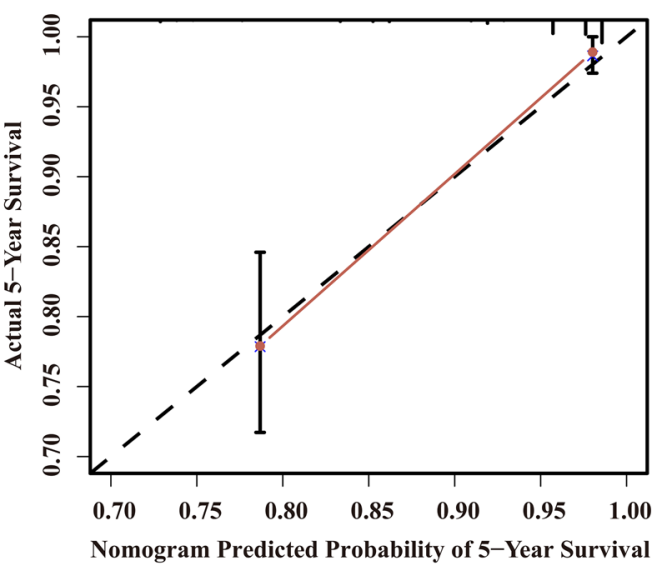

E

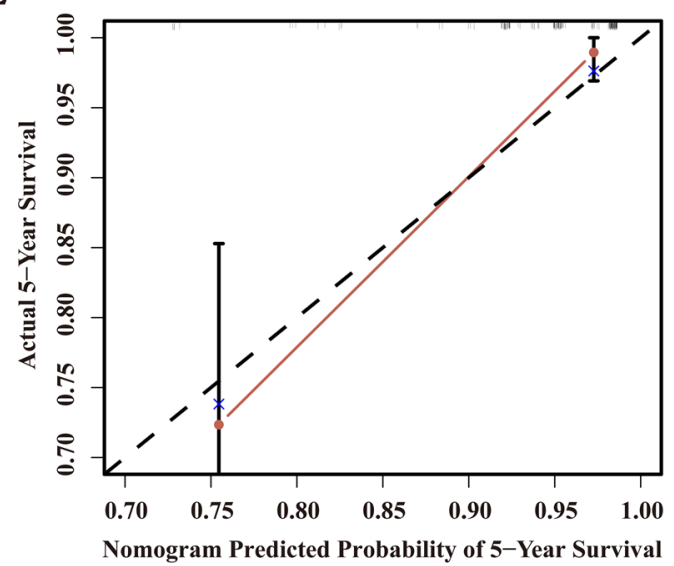

Figure 3: Overall survival predictions based on a nomogram including inflammation-based prognostic scores and clinicopathological characteristics in patients with localized or locally advanced CCRCC. (A) Nomogram for predicting 3- and 5-year OS in primary cohort patients. Calibration curves for predicting 3- (B) and 5-year (C) survival in primary cohort patients. Calibration curves for predicting 3- (D) and 5-year (E) survival in validation cohort patients. 
NLR, and MLR can be determined from routine peripheral blood tests conducted during preoperative examinations. The nomogram used here may therefore serve as a reliable tool for predicting survival in patients with localized or locally advanced CCRCC, as well as for selecting patients for adjuvant therapy trials.

Some limitations should be considered when interpreting the results of this study. First, the relatively small number of patients involved were enrolled at a single institution; the results should therefore be verified in a larger, standardized group of patients. In addition, because this was a retrospective study, there were methodological differences among the studies examined, and a multicenter prospective study should be conducted to confirm our findings.

In conclusion, we evaluated the prognostic value of AGR in patients with localized or locally advanced CCRCC for the first time. AGR was an independent prognostic factor and predicted prognosis more accurately than more established inflammation-based prognostic scores. Finally, the incorporation of AGR with inflammation-based prognostic scores and traditional staging systems in a single nomogram increased its predictive accuracy in these CCRCC patients.

\section{MATERIALS AND METHODS}

\section{Patients}

Written informed consent was obtained from all patients enrolled in this study. The study was approved by the Ethics and Scientific Committees of The Third Affiliated Hospital of Soochow University. Clinicopathological data were collected from 592 patients with RCC who underwent radical or partial nephrectomy in The Third Affiliated Hospital of Soochow University (China) between 2003 and 2013. To ensure that data were collected objectively and accurately, the following exclusion criteria were used: patients with a history of anti-tumor therapy and other concurrent tumors; other acute or chronic concurrent non-cancer diseases (including liver disease, inflammation, and infection); concurrent distant metastasis; patients lost to follow-up. 176 patients who were enrolled between May 2012 and December 2013 were assigned to the external validation cohort; all other patients were assigned to the primary cohort.

\section{Data collection}

The following clinicopathologic data were collected for each enrolled patient: age at surgery, sex, tumor size, Fuhrman grade, and the presence or absence of tumor necrosis, lymph node invasion, and lymphovascular invasion. TNM stage was assigned according to the 2010 AJCC TNM classification [3]. Tumor necrosis was defined as microscopic coagulative necrosis [26]. Lymphovascular invasion was defined as tumor cell invasion of blood vessels or lymphatic vessels, but not the underlying muscular walls [27]. Relevant laboratory indicators and levels of $\mathrm{C}$ - reactive protein (CRP), albumin, globulin, $\mathrm{Hb}$, LDH, etc., were collected one week before surgery. Postoperative follow-ups occurred every six months for the first three years and annually thereafter for locally advanced CCRCC patients. For localized CCRCC patients, followup imaging was performed twice in the first year and annually thereafter. No neoadjuvant or adjuvant treatments were administered. AGR was calculated as AGR = albumin / (total protein - albumin). NLR is the ratio of neutrophils to lymphocytes [21]; MLR is the ratio of monocytes to lymphocytes [22]; and PLR is the ratio of platelets to lymphocytes [23]. GPS was classified as follows: score 2 if serum CRP $>10 \mathrm{mg} / \mathrm{L}$ and albumin $<35 \mathrm{~g} / \mathrm{L}$; score 1 if CRP $>10 \mathrm{mg} / \mathrm{L}$ or albumin $<35 \mathrm{~g} / \mathrm{L}$; score 0 if serum $\mathrm{CRP} \leq 10 \mathrm{mg} / \mathrm{mL}$ and albumin $>35 \mathrm{~g} / \mathrm{L}$ [24]. $\mathrm{mGPS}$ was classified as follows: score 2 if serum CRP $>10 \mathrm{mg} / \mathrm{L}$ and albumin $<35 \mathrm{~g} / \mathrm{L}$; score 1 if serum CRP $>10 \mathrm{mg} / \mathrm{L}$ and albumin $\geq 35 \mathrm{~g} / \mathrm{L}$; score 0 if $\mathrm{CRP} \leq 10 \mathrm{mg} / \mathrm{L}$ [28].

\section{Statistical analysis}

The optimal cut-off values were determined using receiver operating characteristics (ROC) curve analysis. OS rates were calculated using the Kaplan-Meier method and compared to detect statistically significant differences using the log-rank test. PSM was conducted using a nearest-neighbor matching algorithm with a maximum tolerated difference between propensity scores of less than $30 \%$ of the propensity score SD. Mantel-Cox regression methodology was used for univariate analysis of implicit factors affecting survival. Only variables with $P<0.05$ in univariate analyses were included in the multivariate Cox's proportional hazards model. AUC values of the ROC curves were compared to evaluate the discriminatory ability of AGR and the other established inflammationbased prognostic scores in the assessing prognosis. Differences were compared with MedCalc software (Version 11.4.2.0, MedCalc, Inc., Belgium) to determine whether they were statistically significant.

The nomogram was constructed based on the multivariate analysis results. To find a best-fit model, backward stepwise selection with the Akaike information criterion (AIC) was used in a Cox proportional hazards regression model [29]. Both discrimination and calibration were used to evaluate nomogram performance. The c-index and the ROC curves were used to compare OS discrimination ability among different models. Confidence intervals (CIs) were obtained by creating 500 bootstrap samples from the entire data set and replicating the estimation process.

Statistical tests were performed using SPSS software (SPSS 22.0, Chicago, IL, USA), MedCalc software, and R software version 3.2.3 (http://www.r-project.org/) with 
Hmisc, rms, and survival ROC packages. Two-sided $P$ values $<0.05$ were considered statistically significant.

\section{ACKNOWLEDGMENTS AND FUNDING}

This work was supported by the National Science Foundation of Jiangsu Province (Grant no. BK20141161 and Grant no. BK20150251).

\section{CONFLICTS OF INTEREST}

None.

\section{REFERENCES}

1. Mickisch G, Carballido J, Hellsten S, Schulze H, Mensink H. Guidelines on renal cell cancer. Eur Urol. 2001; 40:252-255.

2. Ljungberg B, Campbell SC, Choi HY, Jacqmin D, Lee JE, Weikert S, Kiemeney LA. The epidemiology of renal cell carcinoma. Eur Urol. 2011; 60:615-621.

3. Edge SB, Compton CC. The American Joint Committee on Cancer: the 7th edition of the AJCC cancer staging manual and the future of TNM. Ann Surg Oncol. 2010; 17:1471-1474.

4. Fuhrman SA, Lasky LC, Limas C. Prognostic significance of morphologic parameters in renal cell carcinoma. Am J Surg Pathol. 1982; 6:655-663.

5. Gabay C, Kushner I. Acute-phase proteins and other systemic responses to inflammation. N Engl J Med. 1999; 340:448-454.

6. McMillan DC, Watson WS, O'Gorman P, Preston T, Scott HR, McArdle CS. Albumin concentrations are primarily determined by the body cell mass and the systemic inflammatory response in cancer patients with weight loss. Nutr Cancer. 2001; 39:210-213.

7. Muliawati Y, Haroen H, Rotty LW. Cancer anorexia cachexia syndrome. Acta Med Indones. 2012; 44:154-162.

8. McMillan DC. Systemic inflammation, nutritional status and survival in patients with cancer. Curr Opin Clin Nutr Metab Care. 2009; 12:223-226.

9. Azab BN, Bhatt VR, Vonfrolio S, Bachir R, Rubinshteyn V, Alkaied H, Habeshy A, Patel J, Picon AI, Bloom SW. Value of the pretreatment albumin to globulin ratio in predicting long-term mortality in breast cancer patients. Am J Surg. 2013; 206:764-770.

10. Azab B, Kedia S, Shah N, Vonfrolio S, Lu W, Naboush A, Mohammed F, Bloom SW. The value of the pretreatment albumin/globulin ratio in predicting the long-term survival in colorectal cancer. Int J Colorectal Dis. 2013; 28:1629-1636.

11. Du XJ, Tang LL, Mao YP, Sun Y, Zeng MS, Kang TB, Jia WH, Lin AH, Ma J. The pretreatment albumin to globulin ratio has predictive value for long-term mortality in nasopharyngeal carcinoma. PLoS One. 2014; 9:e94473.

12. Yao Y, Zhao M, Yuan D, Gu X, Liu H, Song Y. Elevated pretreatment serum globulin albumin ratio predicts poor prognosis for advanced non-small cell lung cancer patients. J Thorac Dis. 2014; 6:1261-1270.

13. Leibovich BC, Blute ML, Cheville JC, Lohse CM, Frank I, Kwon ED, Weaver AL, Parker AS, Zincke H. Prediction of progression after radical nephrectomy for patients with clear cell renal cell carcinoma: a stratification tool for prospective clinical trials. Cancer. 2003; 97:1663-1671.

14. Frank I, Blute ML, Cheville JC, Lohse CM, Weaver AL, Zincke H. An outcome prediction model for patients with clear cell renal cell carcinoma treated with radical nephrectomy based on tumor stage, size, grade and necrosis: the SSIGN score. J Urol. 2002; 168:2395-2400.

15. Suh B, Park S, Shin DW, Yun JM, Keam B, Yang HK, Ahn E, Lee H, Park JH, Cho B. Low albumin-to-globulin ratio associated with cancer incidence and mortality in generally healthy adults. Ann Oncol. 2014; 25:2260-2266.

16. Mantovani A, Allavena P, Sica A, Balkwill F. Cancer-related inflammation. Nature. 2008; 454:436-444.

17. Elinav E, Nowarski R, Thaiss CA, Hu B, Jin C, Flavell RA. Inflammation-induced cancer: crosstalk between tumours, immune cells and microorganisms. Nat Rev Cancer. 2013; 13:759-771.

18. Balkwill F, Mantovani A. Inflammation and cancer: back to Virchow? Lancet. 2001; 357:539-545.

19. Onate-Ocana LF, Aiello-Crocifoglio V, Gallardo-Rincon D, Herrera-Goepfert R, Brom-Valladares R, Carrillo JF, Cervera E, Mohar-Betancourt A. Serum albumin as a significant prognostic factor for patients with gastric carcinoma. Ann Surg Oncol. 2007; 14:381-389.

20. Chojkier M. Inhibition of albumin synthesis in chronic diseases: molecular mechanisms. J Clin Gastroenterol. 2005; 39:S143-146.

21. Pichler M, Hutterer GC, Stoeckigt C, Chromecki TF, Stojakovic T, Golbeck S, Eberhard K, Gerger A, Mannweiler S, Pummer K, Zigeuner R. Validation of the pre-treatment neutrophil-lymphocyte ratio as a prognostic factor in a large European cohort of renal cell carcinoma patients. Br J Cancer. 2013; 108:901-907.

22. Chang Y, An H, Xu L, Zhu Y, Yang Y, Lin Z, Xu J. Systemic inflammation score predicts postoperative prognosis of patients with clear-cell renal cell carcinoma. Br J Cancer. 2015; 113:626-633.

23. Gunduz S, Mutlu H, Tural D, Yildiz O, Uysal M, Coskun HS, Bozcuk H. Platelet to lymphocyte ratio as a new prognostic for patients with metastatic renal cell cancer. Asia Pac J Clin Oncol. 2015.

24. Ramsey S, Lamb GW, Aitchison M, Graham J, McMillan DC. Evaluation of an inflammation-based prognostic score in patients with metastatic renal cancer. Cancer. 2007; 109:205-212.

25. Lamb GW, Aitchison M, Ramsey S, Housley SL, McMillan DC. Clinical utility of the Glasgow Prognostic Score in patients undergoing curative nephrectomy for renal clear cell cancer: basis of new prognostic scoring systems. Br J Cancer. 2012; 106:279-283. 
26. Ito K, Seguchi K, Shimazaki H, Takahashi E, Tasaki S, Kuroda K, Sato A, Asakuma J, Horiguchi A, Asano T. Tumor necrosis is a strong predictor for recurrence in patients with pathological T1a renal cell carcinoma. Oncol Lett. 2015; 9:125-130.

27. Katz MD, Serrano MF, Humphrey PA, Grubb RL 3rd, Skolarus TA, Gao F, Kibel AS. The role of lymphovascular space invasion in renal cell carcinoma as a prognostic marker of survival after curative resection. Urol Oncol. 2011; 29:738-744.
28. Proctor MJ, Morrison DS, Talwar D, Balmer SM, O'Reilly DS, Foulis AK, Horgan PG, McMillan DC. An inflammation-based prognostic score (mGPS) predicts cancer survival independent of tumour site: a Glasgow Inflammation Outcome Study. Br J Cancer. 2011; 104:726-734.

29. Harrell FE Jr, Lee KL, Mark DB. Multivariable prognostic models: issues in developing models, evaluating assumptions and adequacy, and measuring and reducing errors. Stat Med. 1996; 15:361-387. 